\title{
Efficacy and Safety of Intravenous Urokinase and Sodium Ozagrel in Acute Ischemic Stroke
}

\author{
Yuan Nong ${ }^{1,}$, Bernard Poon-Lap CHAN ${ }^{2}$, Xingyue Qin ${ }^{1}$, Chong Wei ${ }^{1}$, Kui Chen ${ }^{1}$, Hai Xiao ${ }^{1,}$ * \\ ${ }^{1}$ Department of Neurology, Guigang City People's Hospital, The Eighth Affiliated Hospital of Guangxi Medical University, Guigang, China \\ ${ }^{2}$ Division of Neurology, National University Hospital, Singapore
}

\section{Email address:}

noyu219828@163.com (Yuan Nong),XH13635012077@163.com (Hai Xiao)

*Corresponding author

\section{To cite this article:}

Yuan Nong, Bernard Poon-Lap. CHAN, Xingyue Qin, Chong Wei, Kui Chen, Hai Xiao. Efficacy and Safety of Intravenous Urokinase and Sodium Ozagrel in Acute Ischemic Stroke. American Journal of Psychiatry and Neuroscience. Vol. 6, No. 2, 2018, pp. 51-55. doi: 10.11648/j.ajpn.20180602.14

Received: June 12, 2018; Accepted: July 2, 2018; Published: July 30, 2018

\begin{abstract}
Urokinase and sodium ozagrel are widely used in patients with acute ischemic stroke (AIS) in China. But the effectiveness and safety of the two combinations are not yet clear. A total of 129 AIS patients who were treated with combined intravenous (IV) urokinase and sodium ozagrel within 6 hours of onset were included in this study. All the patients were assessed with the National Institute of Health Stroke Scale (NIHSS) score at baseline, 6 hours, at hospital discharge, and 1 month after AIS. All the patients were characterized into two groups based on early response (decrease in NIHSS score $\geq 4$ points at 6 hours) and good outcome (NIHSS score $\leq 1$ at 1 month), and assessed treatment safety by evaluating intracranial hemorrhage and mortality. There were 54 patients in the good outcome group and 74 in the bad outcome group at the end. Multivariate analysis showed that shorter onset to treatment time, a lower baseline NIHSS score, and lack of large artery stenosis or occlusion werel associated with good outcome at 1 month. This study suggested that combined IV urokinase and sodium ozagrel therapy was effective and safe in treating patient with AIS within a 6-hour time window. With lower cost and a longer time window, it can be used as an alternative intravenous thrombolytic therapy in patients with AIS except rt-PA.
\end{abstract}

Keywords: Urokinase, Ozagrel, Acute Ischemic Stroke, Effective, Safety

\section{Introduction}

The effectiveness of intravenous thrombolysis for the treatment of cerebral infarction in the acute stage has been widely confirmed [1-4]. At present, recombinant tissue plasminogen activator (rt-PA) is the only thrombolytic agent approved by the Food and Drug Administration (FDA), and has been widely used in treating acute ischemic stroke within 4.5 hours of onset [5-7]. However, in some developing countries, the use of rt-PA in patients with acute ischemic stroke is extremely low, often due to high price, late hospital arrival, or limited facilities such as CT scan facility or stroke unit $[8,9]$.

In China, the use of urokinase, an alternative thrombolysis agent, is common given its cheaper cost (about 7 dollars/0.1 million $U$ in China) and longer window time (within 6 hours of onset). Urokinase is an enzyme isolated from healthy human urine and acts directly on the endogenous fibrinolytic system. It catalyzes plasminogen into its active form, plasmin, a fibrinolytic enzyme that degrades fibrin clots, and cleaves and inactivates the coagulation factors V and factor VIII, thus achieving a thrombolytic effect. Although urokinase has multiple anti-clotting effects, its plasma half-life is shorter than 20 minutes. When administered intravenously, its efficacy is significantly weakened once it reaches the intracranial artery, leading to a low vascular thrombolysis recanalization rate. Therefore, single agent treatment with urokinase is not an ideal effective treatment.

Sodium ozagrel is a thromboxane A2 synthetase inhibitor used widely in China that inhibits platelet aggregation and may inhibit thrombosis. In our hospital, we found that intravenous (IV) sodium ozagrel and urokinase enhanced the thrombolytic effect of urokinase, increased the recanalization rate, and resulted in a low incidence of bleeding. Therefore, combination IV urokinase and sodium ozagrel therapy may be 
a useful therapy for acute cerebral infarction.

\section{Method}

\subsection{Materials}

Patients with acute ischemic stroke who were treated with IV urokinase and sodium ozagrel in our hospital neurology department from January 2012 to July 2016 were included in this study. All patients met the following conditions: (1) onset to treatment $<6.0$ hours; (2) computerized tomography (CT) scan either without demonstrated an early low density lesion related to acute infarction nor without hemorrhage; (3) consciousness or lethargy; (4) no acute myocardial infarction or acute stroke in the past 3 months; (5) normal platelet and coagulation function; (6) blood pressure within 180/100 $\mathrm{mmHg}$ before starting treatment; (7) baseline National Institutes of Health Stroke Scale (NIHSS) score $\geq 4$ and $<25$; and (8) a signed informed consent form. We excluded patients with the following conditions: (1) intracranial hemorrhage history; (2) bleeding tendency and bleeding illness; (3) severe diabetes, cardiac insufficiency, or hepatic and renal illness; (4) anticoagulant therapy received in the past 48 hours; or (5) pregnancy.

This study was approved by the Guigang City People's Hospital ethics committee and all patients or their families gave their informed consent.

\subsection{Methods}

All the patients were measured blood pressure, performed routine blood tests, blood sugar tests, and blood lipid examination, measured coagulation function, took a noncontrast CT scan of the head, evaluated NIHSS score, and performed an electrocardiogram (ECG) when they were admitted to the hospitall within one hour. Following IV urokinase treatment, the noncontrast CT scan of head was reviewed in 6 hours. Within 72 hours, all the patients were performed a neck vascular ultrasound, transcranial Doppler and/or magnetic resonance imaging (MRI) scan, and a magnetic resonance or CT angiography of the head.

At the start of treatment, 0.7-1.5 million units of urokinase were added to $100 \mathrm{ml}$ normal saline and intravenously infused within 30 minutes. Following this, $80 \mathrm{mg}$ of sodium ozagrel were added to $250 \mathrm{ml}$ normal saline and intravenously infused at a rate of 40-60 drops per minute. When the head CT was reviewed and hemorrhaging was confirmed to be absent, sodium ozagrel was given two times daily for seven days, or until discharge. Traditional Chinese medicine treatments, including acupuncture or Chinese patent medicine prepared for IV administration were also used in the patients during hospitalization.

A neurologist assessed patient NIHSS score at baseline, 6 hours after admission to the hospital, at discharge, and at 1 month. All clinical data such as gender, age, vascular risk factors, blood pressure, onset to treatment time, NIHSS scores, large artery stenosis or occlusion, and brain CT scan results were registered. Large artery refers to the common carotid artery, internal carotid artery, middle cerebral artery, vertebral artery, and basilar artery in this study. Artery stenosis was defined as a vascular stenosis rate $\geq 50 \%$ compared with the proximal artery.

The absolute change of the NIHSS score 6 hours after admission and at 1 month were calculated. Early response was defined as decreased NIHSS score $\geq 4$ at 6 hours, and a good outcome was defined by a NIHSS score $\leq 1$ at 1 month. The safety of IV urokinase and sodium ozagrel was assessed by the rate of intracranial hemorrhage and mortality.

\subsection{Statistical Analysis}

Patients were divided into either a good outcome group or a bad outcome group according to patient characteristics at 1 month. To determine whether any variables were predictive of good outcome, the general clinical characteristics were compared between the two groups. Variables such as age, onset to treatment time, and NIHSS score were expressed as the mean \pm standard deviation or by the median (range). Noncontinuous variables were presented as a percentage. Multivariate logistic regression analysis was performed to identify predictive variables of good outcomes at 1 month. The SPSS 17.0 statistic package was used for all statistical analyses and a statistically significant difference was defined as $P<0.05$.

\section{Results}

A total of 1802 patients with acute cerebral infarction were registered in our hospital during the period between January 2012 and July 2016. One hundred twenty-nine of the total 1802 patients $(7.2 \%)$ were treated with IV urokinase and sodium ozagrel. Of the 129 patients, 77 were male and 56 were female and the mean age was $64.1( \pm 11.1$ years, range 40-85 years). Patients remained in the hospital for an average of 10-11 days (10.8 7.7 days). This study found that the median NIHSS score decreased over the course of the study, from admission to 1 month. Importantly, early response occurred in almost half of patients (46.5\%), and classified $41.9 \%$ of patients in as having a good outcome. Intracranial hemorrhage was observed in a small percentage of patients: $6.2 \%$ with symptomatic intracranial hemorrhage (ASICH), and $2.3 \%$ with symptomatic intracranial hemorrhage $(\mathrm{SICH})$. During the course of the study, 3 patients died, including 2 patients with SICH and 1 who experienced neurological deterioration and ceased treatment after 2 days.

To determine whether there were any predictive factors for good or bad outcomes in these patients, the clinical characteristics were compared for each group (Table 1). Of all factors, onset to treatment time, baseline NIHSS score, the percentage of large artery stenosis or occlusion, and length of hospital stay were significantly different between the good outcome group and bad outcome group. In the multivariate logistic regression analysis, only onset to treatment time, baseline NIHSS score, and large artery stenosis or occlusion were independent predictive factors of good outcome at 1 month (Table 2). 
Figure 1. shows the clinical outcome and NIHSS score for all patients at 1 month. We found that mild neurological deficit or complete resolution of the neurological deficit occurred in 77 patients at this time.

Table 1. General clinical characteristics of acute ischemic stroke patients treated with urokinase and sodium ozagrel compared by patient outcome.

\begin{tabular}{llll}
\hline Variable & Good outcome group (n=54) & Bad outcome group (n=75) & P value \\
\hline Sex (male:female) & $26: 28$ & $51: 24$ & 0.023 \\
Age & $64.17 \pm 11.49$ & $63.99 \pm 10.89$ & 0.928 \\
Stroke history (\%) & 14.8 & 10.7 & 0.481 \\
Onset to treatment time (h) & $2.8 \pm 1.1$ & $3.3 \pm 1.1$ & 0.010 \\
Urokinase dose (million units) & $1.03 \pm 0.12$ & $1.07 \pm 0.14$ & 0.091 \\
Baseline NIHSS score & $10.2 \pm 2.4$ & $12.1 \pm 3.3$ & 0.001 \\
Pretreatment MAP (mmHg) & $115.1 \pm 12.8$ & $112.5 \pm 13.1$ \\
Pretreatment blood sugar (mmol/L) & $7.0 \pm 2.9$ & $6.8 \pm 2.4$ & 0.276 \\
Pretreatment LDL-C & $3.4 \pm 1.0$ & $3.1 \pm 0.9$ & 0.565 \\
Large artery stenosis/occlusion (\%) & 18.5 & 46.7 & 0.052 \\
Atrial fibrillation (\%) & 5.6 & 5.3 & 0.001 \\
Hospital stay (days) & $8.9 \pm 5.1$ & $12.2 \pm 8.9$ \\
NSICH & 0 & 8 \\
SICH & 0 & 3 \\
Death & 0 & 3 \\
\hline
\end{tabular}

Table 2. Variables associated with good outcome (NIHSS score $\leq 1)$ at 1 month using multivariate logistic regression.

\begin{tabular}{llll}
\hline Variable & B & OR & 95\% CI \\
\hline Onset to treatment time & 0.697 & 2.008 & $1.342-3.004$ \\
Baseline NIHSS score & 0.221 & 1.247 & $1.063-1.464$ \\
Large artery stenosis/occlusion & -1.419 & 0.242 & $0.092-0.639$ \\
\hline
\end{tabular}

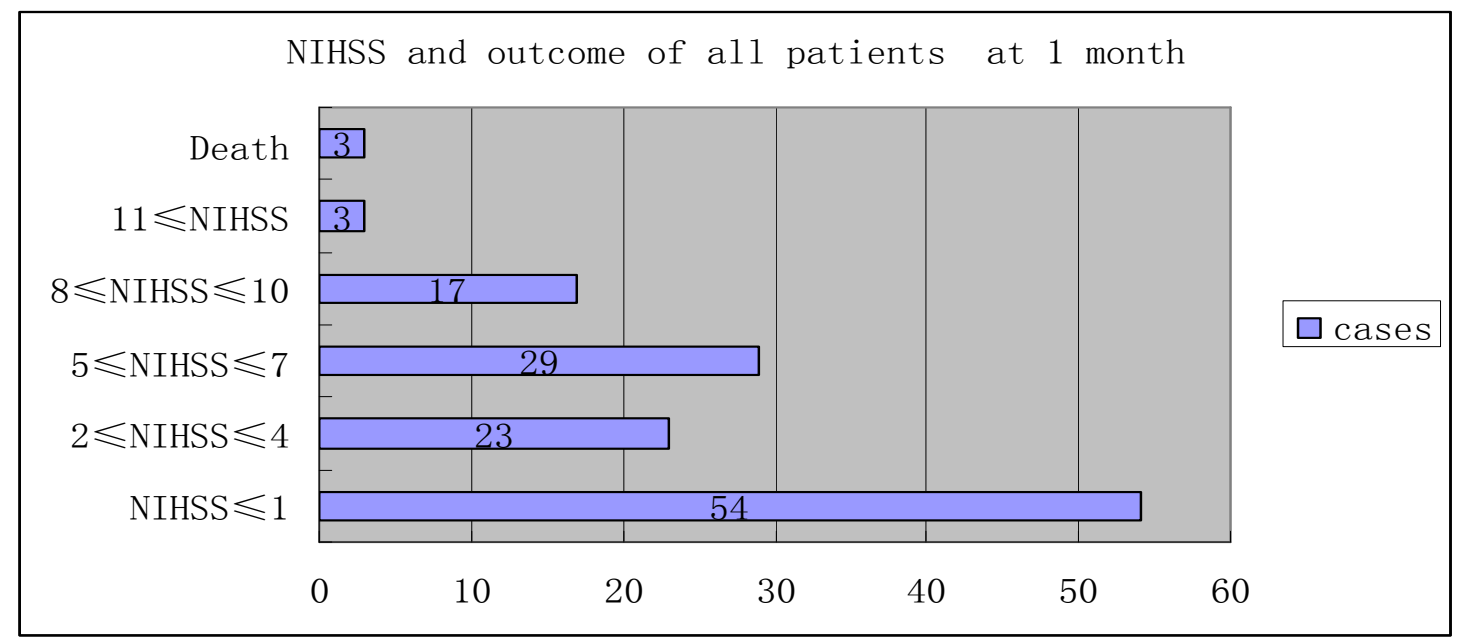

Figure 1. NIHSS score and outcome of all patients at 1 month.

\section{Discussion}

Urokinase for acute ischemic stroke is only used in China, and combination therapy with sodium ozagrel is rare. Combined IV urokinase and sodium ozagrel therapy is reported to increase the chances of hemorrhage. However, in this study, only $2.3 \%$ patients developed $\mathrm{SICH}$, a rate similar to or less than other studies that examined the efficacy of rt-PA $[1,2,5,10,11]$. Additionally, only $2.3 \%$ mortality was observed in this study, which is low $[1,2,9,12]$.

Owing to lack of human resources, funding, and lower patient compliance, it were unable to obtain the standard outcome judgment with modified Rankin scale (mRS) at 3 months in this study. All patients were followed up to 1 month and only recorded their NIHSS score. In this study, the efficacy of treatment was evaluated by defining a good outcome of a NIHSS score $\leq 1$ at 1 month. In the National Institute of Neurological Disorders and Stroke (NINDS) study [1], $39 \%$ patients reached $\mathrm{mRS} \leq 1$, which was equal to the $31 \%$ that had a NIHSS score $\leq 1$ at 3 months. In this study, 54 patients $(41.9 \%)$ were found had a NIHSS score $\leq 1$ at 1 month, and $23(17.8 \%)$ had a NIHSS score of 2-4. If the follow-up time was sufficient, the 23 patients may had more better NIHSS score at 3 months. So, it was might expect about $52.6 \%-75.1 \%$ patients might reach a mRS of $0-1$ at 3 months. Together, regardless of the short follow-up, this study indicates that combined IV urokinase and sodium ozagrel is safe and effective for treating acute ischemic stroke.

The safety and efficacy of intravenous thrombolytic therapy in patients greater than 80 years of age has been confirmed by 
multiple studies [13-16]. There were 12 elderly acute ischemic stroke patients (age $\geq 80$ years) in this study that received therapy, but 2 of the 12 patients developed SICH and died. The sample size was too small to confirm the safety of urokinase and sodium ozagrel treatment in the elderly, and it would still recommend caution in this patient population.

The therapeutic window of urokinase is within 6 hours of stroke onset, and it was found that urokinase effectiveness was influenced by the length of onset to treatment time. The onset-to treatment time for the good outcome group $(2.8 \pm 1.1$ hours) was significantly shorter than that of the bad outcome group $(3.3 \pm 1.1$ hours $)(P<0.05)$. Furthermore, onset to treatment time was an independently correlated with good outcome at 1 month $(\mathrm{OR}=2.008,95 \% \mathrm{CI}=1.342-3.004)$. But it was not easy in China to get a shorter onset to treatment time, multiple factors influence this time window including: lack of education about stroke symptoms, apprehension of the adverse effects of thrombolysis [9], lack of trust in doctors and medical staff, traffic, and abuse and lack of emergency resources.

In this study, it was found that baseline NIHSS score and large artery stenosis or occlusion were related to patient outcomes at 1 month. The patients with a lower baseline NIHSS score $(\mathrm{OR}=1.247,95 \% \mathrm{CI}=1.063-1.464)$ without large artery stenosis or occlusion $(\mathrm{OR}=0.242,95 \% \mathrm{CI}=0.092-0.639)$ improved at the 1-month time point in this study. A lower NIHSS score and lack of large artery stenosis or occlusion are associated with fewer neurological functional defects, and good cerebral blood circulation, respectively. Therefore, these factors suggest the possibility of a larger stroke occurring is small and the patients with these characteristics will have favorable outcomes. Furthermore, all cerebral vascular examinations were performed after thrombolytic treatment; therefore, when the patients without large artery stenosis or occlusion in the CTA or MRA, there may be also means that urokinase combined with ozagrel treatment has achieved recanalization in these patients.

There are some limitations in this study. First, the safest and most effective urokinase dose has not yet been established; here, the patients were used 0.7-1.5 million units urokinase according to the doctor's personal experience. Second, the traditional Chinese medicine treatment methods were used in the patients after thrombolysis therapy. While, the mechanism of traditional Chinese medicine for treatment of stroke is not clear; therefore, it cannot determine whether a good outcome was in part due to the effect of this treatment. Third, due to lack of funding and staffing, the follow-up time was shorter than other studies [7, 8]. Fourth, some patients in our study did not have acute cerebral infarction lesions following MRI examination after completion of thrombolytic therapy, suggesting that it may have included some transient ischemic attack patients in the effective group, to lead a higher rate of good outcome. Finally, this study is a single-center study with a relatively small sample size. Therefore, multi-center studies with large sample sizes are needed to confirm our result.

\section{Conclusion}

In conclusion, this study shows that IV urokinase combined with sodium ozagrel is effective and safe for treating acute ischemic stroke. With lower cost and a longer time window than rt-PA, it maybe another choice for the AIS patients in developing areas. Meanwhile, a randomized trial to compare urokinase with both the standard and low doses of rt-PA is warranted. In addition, the role and mechanism of sodium ozagrel in acute ischemic treatment should be further investigated.

\section{Conflict of Interest}

All the authors do not have any possible conflicts of interest.

\section{References}

[1] National Institute of Neurological Disorders and Stroke rt-PA Stroke Study Group. Tissue plasminogen activator for acute ischemic stroke. N Engl J Med 1995; 333: 1581-1587.

[2] Tomsick TA. Intravenous thrombolysis for acute ischemic stroke. J Vasc Interv Radiol. 2004; 15: S67-76.

[3] Cronin CA. Intravenous tissue plasminogen activator for stroke a review of the ECASS III results in relation to prior clinical trials. J Emerg Med. 2010; 38: 99-105.

[4] Hatcher MA, Starr JA. Role of tissue plasminogen activator in acute ischemic stroke. Ann Pharmacother. 2011; 45: 364-371.

[5] Hacke W, Kaste M, Bluhmki E, et al. Thrombolysis with alteplase 3 to 4.5 hours after acute ischemic stroke. N Engl J Med. 2008; 359: 1317-1329.

[6] Ahmed N, Wahlgren N, Grond M, et al. Implementation and outcome of thrombolysis with alteplase 3-4.5 hours after an acute stroke: an updated analysis from SITS-ISTR. Lancet Neurol 2010; 9: 866-874.

[7] Shobha N, Buchan AM, Hill MD, et al. Thrombolysis at 3-4.5 hours after acute ischemic stroke onset--evidence from the Canadian Alteplase for Stroke Effectiveness Study (CASES) registry. Cerebrovasc Dis. 2011; 31: 223-228

[8] Ghandehari K. Barriers of thrombolysis therapy in developing countries. Stroke Res Tre. 2011; 2011: 686797. doi: 10. 4061/2011/686797. Epub 2011 Mar 17.

[9] Wang Y, Wu D, Zhao X, et al. Hospital resource for urokinase/ recombinant tissue-type plasminogen activator therapy for acute stroke in Beijing. Surg Neurol 2009; 72 (Suppl. I): 2-7.

[10] $\mathrm{Xu} \mathrm{ZP,} \mathrm{Li} \mathrm{HH,} \mathrm{Li} \mathrm{YH,} \mathrm{et} \mathrm{al.} \mathrm{Feasibility} \mathrm{and} \mathrm{outcomes} \mathrm{of}$ intravenous thrombolysis 3-4.5 hours after stroke in Chinese patients. J Clin Neurosci. 2014; 21: 822-826.

[11] Sadeghi-Hokmabadi E, Farhoudi M, Taheraghdam A, et al. Intravenous recombinant tissue plasminogen activator for acute ischemic stroke: a feasibility and safety study. Int J Gen Med 2016; 9: 361-367.

[12] Sharma VK, Tsivgoulis G, Tan JH, et al. Feasibility and safety of intravenous thrombolysis in multiethnic Asian stroke patients in Singapore. J Stroke Cerebrovasc Dis. 2010; 19: 424-430. 
[13] Simon JE, Sandler DL, Pexman JHW, HillMD, Buchan AM: Is intravenous recombinant tissue plasminogen activator (rt-PA) safe for use in patients over 80 years old withacute ischaemic stroke? - the Calgary expe-rience. Age Ageing 2004; 33: 143-149.

[14] Zeevi N, Chhabra J, Silverman IE, et al. Acute stroke management in the elderly. Cerebrovasc Dis. 2007: 23: 304-308.
[15] Chen Y, Li CH, Wang YX, et al. Safety and effectiveness of intravenous thrombolysis with recombinant tissue plasminogen activator in eighty years and older acute ischemic stroke patients. Eur Rev Med Pharmacol Sci. 2015; 19: 1852-1858.

[16] Reuter B, Gumbinger C, Sauer T. et al. Intravenous thrombolysis for acute ischaemic stroke in the elderly: data from the Baden-Wuerttemberg stroke registry. Eur J Neurol. 2016; 23: 13-20. 